\title{
To study the efficacy of screening panel including interleukin-6 in diagnosis of neonatal sepsis
}

\author{
Archana Dubey ${ }^{1}$, Sagar Narang ${ }^{2 *}$, Anita Pandey ${ }^{3}$, Vandana Singh ${ }^{4}$, Devyani Sharma ${ }^{5}$ \\ ${ }^{\mathbf{1}}$ Professor, ${ }^{\mathbf{2}}$ Assistant Professor, ${ }^{3}$ Professor and Head, ${ }^{\mathbf{4}}$ Assistatn Professor, ${ }^{\mathbf{5}}$ Medical Officer, ${ }^{\mathbf{1 , 2 , 4 , 5}}$ Dept. of Pediatrics, ${ }^{\mathbf{3}}$ Dept. of \\ Microbiology, ${ }^{1-4}$ Subharti Medical College, Meerut, Uttar Pradesh, ${ }^{5}$ Sai Hospital, Meerut, Uttar Pradesh, India \\ *Corresponding Author: Sagar Narang \\ Email: sarang_medical@yahoo.co
}

\begin{abstract}
Objective: To Study the Efficacy of Screening Panel, Including Interleukin 6, in Diagnosis of Neonatal Sepsis.

Materials and Methods: This prospective study was done from 2015-2016 in the Post Graduate department of Pediatrics of Subharti Medical College, Meerut. 100 neonates below the age of 28 days with a clinical suspicion of neonatal sepsis were enrolled in the study. Septic panel (WBC, ANC, I/T ratio, Micro ESR, CRP), IL-6 and blood culture were sent for screening. Data was collected and analyzed in babies having a positive blood culture. Septic panel in blood culture positive babies with $\geq 3$ variables of septic panel without IL-6, were compared with septic panel in blood culture positive babies with $\geq 3$ variables of septic panel with IL-6. Data was analyzed.

Result: Presence of the new marker (IL-6) with the septic panel shows a sensitivity of $65.71 \%$, specificity of $96.92 \%$ and PPV of $92.0 \%$ against the septic panel without IL-6, which shows a sensitivity of $34.28 \%$, specificity of $93.84 \%$ and PPV of $75.0 \%$.
\end{abstract}

Conclusion: Septic panel with IL-6 has more sensitivity, specificity and positive predictive value as compared to septic panel without IL-6.

Keywords: Neonatal sepsis, Interleukin-6.

\section{Introduction}

Neonatal sepsis is defined as an invasive bacterial infection which occurs in the first 4 weeks of life. It is the commonest cause of neonatal mortality and is responsible for $30-50 \%$ of all neonatal deaths each year in developing countries. ${ }^{1-3}$

It is estimated that up to $20 \%$ of neonates develop sepsis and approximately $1 \%$ die of sepsis related causes. ${ }^{2}$ The World Health Organization (WHO) estimates that each year 4 million newborns, worldwide, die during the neonatal period. ${ }^{2}$ Seventy-five percent of these deaths occur during the first week of life. About $25 \%$ to $45 \%$ neonatal deaths occur on the first day of life. ${ }^{3-5}$ In India the incidence of neonatal sepsis varies from 11 - 24.5/ 1000 live births.

The presenting features of sepsis in neonates are nonspecific and subtle, leading to a delay in diagnosis and treatment, there by worsening morbidity and mortality. ${ }^{6}$ Traditional septic screen does not have high specificity or sensitivity to detect sepsis in neonates; thus a new early septic marker (IL-6) was included in the septic screen panel anticipating that the sensitivity and specificity of the septic panel would increase.

\section{Objective}

To study the efficacy of screening panel including interleukin 6 in diagnosis of neonatal sepsis.

\section{Materials and Methods \\ Material}

This prospective study was conducted in a tertiary care centre, Subharti Medical College, Meerut, over a periodofone year 2015-2016. A hundred neonates above 34 weeks gestation and below the age of 28 days admitted in the NICU or Nursery with clinical suspicion of neonatal septicaemia were included in the study.

\section{Method}

Blood was collected with all aseptic precautions and sent for estimation of total leukocyte count, absolute neutrophil count, I/T ratio, another sample was centrifuged and serum collected and preserved in a sterile container and refrigerated at $-20^{\circ} \mathrm{c}$ for IL 6 estimation, a third sample was sent for blood culture.

IL-6 was be detected using a commercial ELISA kit provided by Diaclone and the cut off values were taken as $24.5 \mathrm{pg} / \mathrm{ml}^{7}$

Absolute Neutrophil count was calculated and Micro ESR was noted.

The reports were collected and tabulated.

\section{Observations and Results}

The following statistics can be defined:

1. Sensitivity: probability that a test result will be positive when the disease is present (true positive rate, expressed as a percentage $).=\mathrm{a} /(\mathrm{a}+\mathrm{b})$

2. Specificity: probability that a test result will be negative when the disease is not present (true negative rate, expressed as a percentage $).=d /(c+d)$

3. Positive predictive value: probability that the disease is present when the test is positive (expressed as a percentage $).=\mathrm{a} /(\mathrm{a}+\mathrm{c})$

4. Negative predictive value: probability that the disease is not present when the test is negative (expressed as a percentage $).=d /(b+d)$ 
Table 1: Diagnosticstatistics

\begin{tabular}{|c|c|c|c|c|c|}
\hline Test & Disease Present & N & Disease Absent & n & Total \\
\hline Positive & True Positive & $a$ & False Positive & $c$ & $a+c$ \\
\hline Negative & False Negative & $b$ & True Negative & $d$ & $b+d$ \\
\hline Total & & $a+b$ & & $c+d$ & \\
\hline
\end{tabular}

\section{Significant Figures}

Suggestive Significance $(\mathrm{P}$ value: $0.05<\mathrm{P}<0.10)$

Moderately Significant ( $\mathrm{P}$ value: $0.01<\mathrm{P} \leq 0.05$ )

Strongly Significant (P value: $\mathrm{P} \leq 0.01$ )

100 newborns with probable sepsis were studied.

Table 2: Particulars of the 100 neonates studied who had clinical suspicion of septicemia

\begin{tabular}{|l|c|}
\hline Neonates characteristics & $\mathbf{N = 1 0 0}$ No of neonates \\
\hline Age in days & 84 \\
\hline $0-3$ days & 16 \\
\hline $4-28$ days & \\
\hline Gender & 57 \\
\hline Male & 43 \\
\hline Female & \\
\hline Gestational age & 70 \\
\hline Preterm & 30 \\
\hline Term & \\
\hline Birth weight (gms) & 10 \\
\hline$<1500$ & 60 \\
\hline $1500-2500$ & 30 \\
\hline$>2500$ & \\
\hline Place of birth & 36 \\
\hline Intramural & 64 \\
\hline Extramural & \\
\hline
\end{tabular}

Table 3: Blood investigations of neonates with clinical sepsis

\begin{tabular}{|l|c|}
\hline Blood Investigations & N=100 No of neonates \\
\hline WBC count & 20 \\
\hline$<5000 /$ cumm & 80 \\
\hline$\geq 5000 /$ cumm & \\
\hline ANC & 19 \\
\hline$<1750 /$ cumm & 81 \\
\hline$\geq 1750 /$ cumm & \\
\hline Micro ESR at end of $\mathbf{1}^{\text {st }} \mathbf{h r}$ & 25 \\
\hline Positive $\geq 10 \mathrm{~mm}$ & 75 \\
\hline Negative $<10 \mathrm{~mm}$ & \\
\hline I/T ratio & 23 \\
\hline$\geq 0.2$ & 77 \\
\hline$<0.2$ & \\
\hline CRP & 29 \\
\hline Positive $>5 \mathrm{mg} / \mathrm{dl}$ & 71 \\
\hline Negative $<5 \mathrm{mg} / \mathrm{dl}$ & \\
\hline IL 6 & 32 \\
\hline Positive $>24.65 \mathrm{pg} / \mathrm{ml}$ & 68 \\
\hline Negative $<24.65 \mathrm{pg} / \mathrm{ml}$ & \\
\hline
\end{tabular}


Table 4: Correlation of laboratory parameters in relation to blood culture (Growth/ No growth)

\begin{tabular}{|l|c|c|c|c|}
\hline & True positive & $\begin{array}{c}\text { False } \\
\text { Positive }\end{array}$ & $\begin{array}{c}\text { False } \\
\text { Negative }\end{array}$ & $\begin{array}{c}\text { True } \\
\text { Negative }\end{array}$ \\
\hline WBC $<5000$ & 14 & 6 & 21 & 59 \\
\hline ANC $<1750$ & 15 & 4 & 20 & 61 \\
\hline Micro ESR & 15 & 10 & 20 & 55 \\
\hline I /T ratio & 10 & 13 & 25 & 52 \\
\hline CRP & 22 & 7 & 13 & 58 \\
\hline IL -6 & 19 & 13 & 16 & 52 \\
\hline Combinations & & & & \\
\hline CRP+ WBC & 7 & 11 & 28 & 54 \\
\hline CRP+ANC & 9 & 10 & 26 & 55 \\
\hline CRP+MICRO ESR & 10 & 15 & 25 & 50 \\
\hline CRP+ IL-6 & 12 & 16 & 22 & 49 \\
\hline Any Variable $\geq 3$ without IL-6 & 12 & 4 & 23 & 61 \\
\hline Any Variable $\geq 3$ with IL-6 & 23 & 2 & 12 & 63 \\
\hline
\end{tabular}

Table 5: Correlation of WBC Count, ANC, Micro ESR, I/T ratio, CRP, IL-6 and combinations with blood culture (Growth/No growth)

\begin{tabular}{|l|c|c|c|c|c|}
\hline Septic screen parameters & Sensitivity & Specificity & PPV & NPV & Accuracy \\
\hline WBC & 40 & 90.76 & 70 & 73 & 14.59 \\
\hline ANC & 42.85 & 93.84 & 78.94 & 75.30 & 15.61 \\
\hline Micro ESR & 42.85 & 84.61 & 60 & 64.70 & 15.55 \\
\hline I /T ratio & 28.5 & 80 & 43.4 & 67.75 & 10.52 \\
\hline CRP & 62.85 & 89.23 & 75.86 & 81.69 & 22.58 \\
\hline IL-6 & 54.28 & 80 & 59.37 & 76.47 & 19.52 \\
\hline Combinations & & & & & \\
\hline CRP+ WBC & 20 & 83.07 & 38.88 & 65.8 & 7.54 \\
\hline CRP+ANC & 28.71 & 84.61 & 47.36 & 67.90 & 9.55 \\
\hline CRP+MICRO ESR & 28.57 & 76.92 & 40 & 66.66 & 10.5 \\
\hline CRP+ IL-6 & 37.14 & 75.38 & 44.82 & 69.01 & 13.49 \\
\hline Any Variable $\geq 3$ Without IL-6 & 34.28 & 93.84 & 75.0 & 72.61 & 12.61 \\
\hline Any Variable $\geq 3$ With IL-6 & 65.71 & 96.92 & 92.00 & 84.00 & 23.63 \\
\hline
\end{tabular}

\section{Discussion}

Indirect markers of infection when collectively studied provide an extremely reliable index of early suspicion of neonatal sepsis and serve as a useful guide for initiating antibiotic therapy. Bacterial culture positivity gave definitive diagnosis of septicemia.

In this study out of 100 cases of suspected sepsis 35 cases were proven culture positive. It was observed that CRP had highest sensitivity, specificity, PPV and NPV followed by IL-6, micro ESR, absolute neutrophil count (ANC), WBC count and I/T ratio. Combination of septic panel without IL-6 test had a sensitivity of $34.28 \%$, specificity of $93.84 \%$, and positive predictive value of $75 \%$. Septic panel with IL-6 had a sensitivity of $65.71 \%$, specificity of $96.92 \%$, and positive predictive value of $92.0 \%$.

We have made two investigating septic panels (including WBC, ANC, Micro ESR and I/T ratio) which were considered.

1. Septic panel without IL-6

2. Septic panel with IL-6
In the first investigating Septic panel i.e. without IL-6, we observed that the sensitivity was $37.14 \%$, specificity $75 \%$, PPV $44.8 \%$ with significant $p$ value. Taker $\mathrm{R}^{7}$ and Ramesh $\mathrm{Bhat}^{8}$ observed similar results in their studies too. Alistair et $\mathrm{al}^{9}$ and Gerdes et $\mathrm{al}^{10}$ observed high sensitivity and specificity in their studies.

In the second investigating Septic panel i.e. with IL-6, sensitivity was seen to be $65.71 \%$, specificity $96.92 \%$ and PPV 92. All the parameters were increased with addition of IL-6 in the septic panel. In another study by Henrit et al (2001) similar results were obtained as in this study, with a significant $p$ value.

On comparing the two groups we found that all parameters were statistically significant in the group with IL-6.

\section{Summary and Conclusion}

To detect early sepsis in neonates a new early septic marker (IL-6) was included in the septic screen in this study, which detects sepsis even before the appearance of clinical features. Using this early marker it was observed that independently IL-6 was not a good marker but if included in 
the septic panel then the efficacy of the septic panel in terms of sensitivity, specificity and PPV were increased, though the increase was not significant.

This was due to the limitation of this study. Interleukin6 (IL-6) is an early marker of sepsis which becomes positive even before the appearance of clinical features. As the newborn becomes symptomatic the level of IL-6 starts decreasing. To determine the significance of this marker ideally IL-6 levels should be tested hourly in order to detect the decreasing trend.

Moreover till now studies have not able to define any specific normal range for IL-6 for a particular geographic area.

In conclusion this study has limitations and more work is required in this field to make IL-6 a part of routine neonatal septic screening.

\section{Conflict of Interest: None.}

\section{References}

1. Venkatesh M, Merenstein GB, Adams K. Infection in the neonate. Merenstein GB, Gardner SL, editors. Handbook of neonatal intensive care. 6 th edition. St. Louis $(\mathrm{MO})$ : Mosby;2006. p. 569-93.

2. Lawn JE, Cousens S, Zupan J. 4 million neonatal deaths: when? Where? Why? Lancet 2005;365: 891-900.

3. The Young Infants Clinical Signs Study Group. Clinical signs that predict severe illness in children under age 2 months: a multi-centre study. Lancet 2008;371:135-42.

4. Aggarwal R, Sarkar N, Deorari AK. Sepsis in neonate. Indian J Pediatr 2001;58:143-7.
5. Jaiswal RS, Kaushal RK, Goel A, Pathania K. Role of the Creactive protein in deciding the duration of the antibiotic therapy in neonatal septicaemia. Indian Paediatr 2003;40:80083.

6. World Health Organization. World health report 2005: Make every mother and child count. Geneva: World Health Organization; 2006.

7. Taker R. Laboratory Diagnosis of Neonatal Sepsis. Paediatrics Today 2006;9(5):250-5.

8. Ramesh Bhat Y, Kumar N. Outcome of sepsis evaluation in very-low birth- weight premature neonates. J Clin Diagn Res 2009;31847-52.

9. Alistair GS, Philip MB, Jean R. Hewitt BS. Early diagnosis of neonatal sepsis. Pediatr 1980;65(5):1036-41.

10. Gerdes JS, Polin R. Early diagnosis and treatment of neonatal sepsis. Indian J Pediatr 1998;65:63-78.

How to cite this article: Dubey A, Narang S, Pandey A, Singh V, Sharma D. To study the efficacy of screening panel including interleukin-6 in diagnosis of neonatal sepsis. Int J Med Paediatr Oncol 2019;5(2):62-5. 